\title{
Diagnosis of Hearing Loss in Newborns and Infants, Through Objective Audiological Assessment
}

\author{
Bárbara Verrecchia ${ }^{2 *}$ and Vicente Curcio ${ }^{1 *}$ \\ ${ }^{1}$ Principal of Audiology Specialization at UMSA, South America \\ ${ }^{2}$ Audiologist. at UMSA, South America
}

Submission: July 25, 2016; Published: August 05, 2016

*Corresponding author: Bárbara Verrecchia and Vicente Curcio, Department of Audiology, Audiology Specialization at UMSA, Argentina, South America.

\begin{abstract}
This article outlines the importance of objective audiological methods in hearing assessment of infants and newborns. The emphasis placed on updating and improving procedures for infant hearing screening in order to achieve a timely and effective detection, diagnosis and treatment for those children suffering from hearing loss, results in a reduction of time and avoidance of mismatching actions in their psycholinguistic development. This is referred to otoacoustic emissions (OAE) and auditory brainstem responses (ABR), emphasizing their specific applications, advantages, disadvantages and the protocol used in Argentina for early detection.

Keywords: Hearing loss; Otoacoustic emissions; Auditory brainstem response; Screening

Abbreviations: JCIH: Joint Committee on Infant Hearing; CODEPEH: Spanish Commission for Early Detection of Hearing Loss; OAE: Otoacoustic Emissions; ABR: Auditory Brainstem Responses; AABR: Automated Auditory Brainstem Response
\end{abstract}

\section{Introduction}

Hearing loss, including profound deafness, is a treatable entity; but the key of its treatment depends on diagnosing the disease as early as possible. Undetected and untreated hearing impairment in time brings severe consequences for the development of speech and language and therefore mental and social development of a child [1]. The main symptoms of hearing impairment in childhood could be within two groups:

I. The striking unresponsiveness of acoustic stimuli.

II. A development significantly absent or deficient speech.

Therefore, it is extremely important to check the hearing status in all newborns, whether or not risk factors evidence themselves.

The risk factors responsible of hearing loss described in the Joint Committee on Infant Hearing (JCIH), and ratified by the Spanish Commission for Early Detection of Hearing Loss (CODEPEH) are:

a. Family history of sensorineural hearing loss.

b. Infections during pregnancy, such as: toxoplasmosis, rubella, cytomegalovirus, herpes, etc.

c. Craniofacial malformations, including major malformations or dysmorphic consequences affecting the average facial line or structures related to the ear.

\section{d. Weight at time of birth less than 1500 grams. \\ e. Severe hyperbilirubinemia.}

f. Use of ototoxic medication, either administered during pregnancy or after birth (aminoglycosides, diuretics, etc.).

g. Bacterial meningitis at any age.

h. Hypoxic-ischemic attack, at any age, especially at the time of delivery (Apgar score of 0 to 4 in the first minute, or 0 to 6 in the fifth minute).

i. Prolonged mechanical ventilation.

j. Clinical signs or findings suggestive of syndromes associated with deafness.

k. Head injury with loss of consciousness or skull fracture.

l. Neurodegenerative disorders and other neurological diseases associated with seizures.

In addition, from the stage of infant to 3 years of age should be considered the following aspects:

m. Suspicion raised by parents of hearing loss in children.

n. Delayed acquisition of normal for the ratio of overall child development audio-linguistic basis. 
o. Presence of chronic persistent or recurrent acute otitis media [2].

\section{Newborn hearing screening in Argentina}

From birth and within the first month of life, every child needs to be audiologically tested, regardless of whether or not has a history of auditory risk; this is known as Universal Hearing Screening. Law 25.415, entitled National Program of Early Detection and Treatment of Hearing Loss, is described below.

The term universal, implies that the assessment should be performed in all infants, regardless of their history of auditory risk. The exact assessment of hearing is difficult for those who cannot respond to subjective tests either by age or by association with other components; making it necessary in such cases the use of physiological measures considered objective, i.e. that do not require patient cooperation. Currently the most reliable diagnostic tests are: otoacoustic emissions (OAE) and auditory brainstem responses (ABR) [3]. (Figure 1)

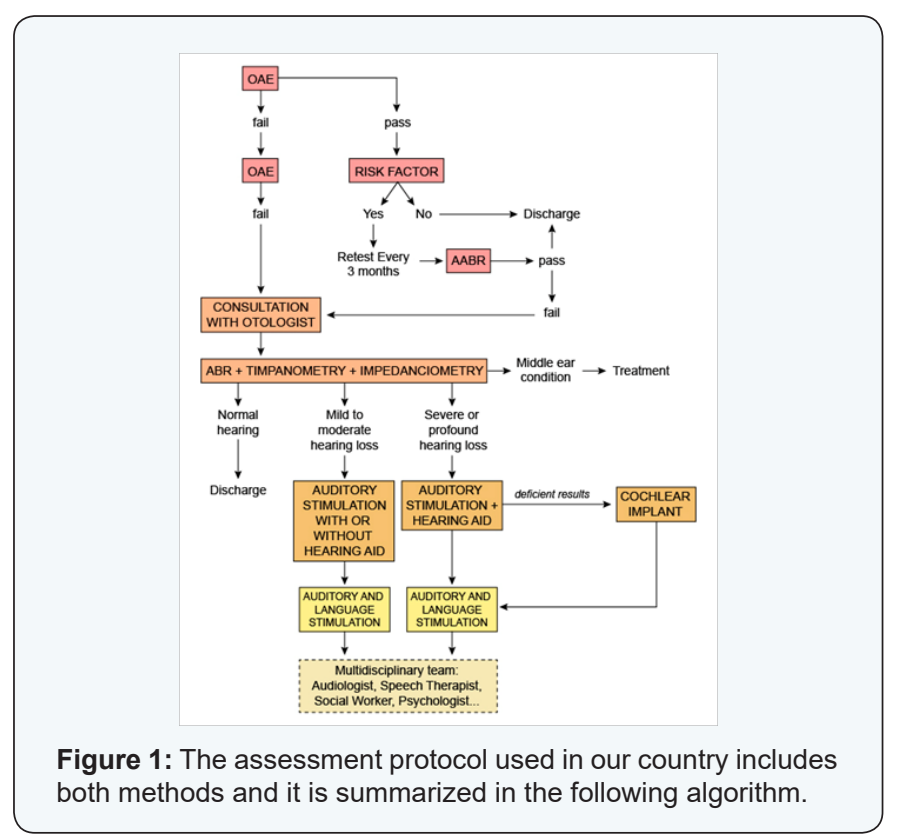

A short summary of these methods will be now described:

Otoacoustic Emissions: Sounds generated in the normal cochlea, either spontaneously or in response to a sound stimulus; reflecting the activity of active biological mechanisms within the inner ear. OAE are responsible for an exquisite sensitivity, a fine frequency selectivity and a wide dynamic range of normal hearing.

Characteristics and qualities that make OAE exceptionally advantageous as clinical trials of cochlear function:

i. $\quad$ bjective

ii. Fast procedure

iii. Accurate

iv. Repeatable v. Noninvasive

vi. Present in normal ears

vii. Absent or reduced in damaged ears [4]

Automated Auditory Brainstem Response (AABR): automatically record the evoked response to the sound stimulus. The presence of a response (pass) or lack there of (refer) to the intensity level of screening, is determined primarily by an algorithm which operates on-line, not professional interpretation being necessary.

Ideal for screening by:

a. Simple application and registration.

b. Fast and reliable procedure.

c. Easy implementation.

d. Objective results (pass and refer).

e. High sensitivity and specificity [5].

Auditory BrainstemResponse to frequency specific stimuli (diagnosis ABR): A complete audiological evaluation of hearing in babies or children must explore frequency specific evoked responses. To achieve this goal we use pip or burst stimuli. These stimuli show good correlation with behavioral responses. Frequencies 500, 1000, 2000 and $4000 \mathrm{~Hz}$ are evaluated with threshold search; thus allowing a full assessment of the degree, type and configuration of hearing loss. In our country, we record auditory brainstem responses under physiological sleep (usually no anesthesia or sedation is used). The interest in the early diagnosis of a hearing loss improves the chances of habilitation and rehabilitation of hearing and speech, with potential is possible to assess the integrity of the auditory pathway from the $25^{\text {th }}$ week of gestation.

Summarizing, diagnosis frequency specific ABR is able to:

a) Evaluate function and pathology of the auditory pathway.

b) Search hearing thresholds.

c) Evaluate development and maturation.

d) Selection of Hearing aid.

e) Easy realization for trained professionals.

f) Not affected by sleep or anesthesia [6].

\section{Conclusion}

A significant number of babies are at risk for hearing loss due to complications related to pre or perinatal issues, family history or other factors occurring at an early age. In order to achieve a proper audiological diagnosis in newborns and infants, we have a wide variety of objective and electrophysiological tests that allows us to take responsibility on this complex task. Hearing loss, including profound deafness, are both treatable entities; but the 
results of treatment depends on diagnosing it as early as possible. Undetected or untreated hearing impairment brings severe consequences for the acquisition of speech and therefore mental and social development of the child [7]. Early diagnosis is the first phase of treatment, because their prognosis improves as early as a therapeutic strategy is established. Early detection programs are key to achieve this goal and are our responsibility as audiologists to be trained and updated on this matter.

\section{References}

1. Katz J (2009) Handbook of clinicalaudiology. Williams and Willkins, UnitedStates, pp. 437-458.

2. Joint Committee on Infant Hearing (2007) Position Statement: Principles and Guidelines for Early Hearing Detection and Intervention Programs. Pediatrics 120(4): 798.
3. Ana Speranza, Nicolás Kreplak, Daniel Gustavo Gollan, Juan Luís Manzur, Cristina Fernández de Kirchner, et al. (2014) Programa Nacional De Fortalecimiento De La Detección Precoz De Enfermedades Congénitas. Pesquisa Neonatal Auditiva

4. Werner A (2006) Teoría y práctica de las otoemisiones acústicas. Edimed, Argentina, USA, pp. 117-135.

5. Hall J (2006) New handbook of auditory evoked responses. Pearson, USA, pp. 320-325.

6. Hood L (1998) Clinical Applications of the Auditory Brainstem Response. Singular Publishing Group, USA, pp. 93-122.

7. Seewald R, Tharpe A (2011) Comprehensive Handbook of Pediatric Audiology. Plural Publishing, Canadá, pp. 283-339. 